\title{
Análisis descriptivo del proceso de cambio tecnológico de sistemas de producción ganadera extensiva a semi intensiva en la Provincia de Espinar, Cusco
}

\section{Descriptive analysis of the process of technological change of extensive livestock production systems to semi intensive in the Province of Espinar, Cusco}

Eibert Sotomayor Obregón ${ }^{1}$, Antonio Salomón Valderrama Romero ${ }^{1,3}$, Edwin Romualdo Benavente Ramírez¹, Jesús Manuel More López ${ }^{2}$, José Yovera Saldarriaga ${ }^{2}$, Ignacio Paucar Meléndez ${ }^{1}, \quad$ Roger Mario Cusihuamán Phocco ${ }^{1}$, Yasmín Jesús Vélez Chang $^{3}$, José Antonio Legua Cárdenas ${ }^{3}$

\section{RESUMEN}

Objetivo: Describir y analizar el proceso de cambio tecnológico en los sistemas de producción ganadera extensiva a semi intensiva en la comunidad campesina de Huisa Ccollana. Los objetivos específicos fueron: Identificar y caracterizar los diferentes sistemas de producción ganadera en la comunidad campesina de Huisa Ccollana; identificar y analizar los factores internos y externos que influyen positiva y/o negativamente en el cambio tecnológico en los sistemas de producción ganadera; identificar y analizar el proceso del cambio tecnológico en los sistemas de producción ganadera. Materiales y Métodos: Para el análisis descriptivo del Cambio Tecnológico se utilizaron los siguientes parámetros de evaluación: Observación, Entrevista semi-estructurada y Encuesta aplicada (mediante la aplicación de una encuesta se pudo recoger datos e información más precisa). La unidad de análisis es la familia campesina (unidad económica familiar), el número de muestras fueron 30 familias, lo cual representó el $25 \%$ del total de familias de la comunidad. El porcentaje de aplicación de tecnologías por sistemas de producción se determinó mediante su evolución por actividad (mejoramiento, alimentación, manejo, sanidad, infraestructura, agroindustria, medio ambiente). Resultados: En la introducción y adopción de tecnologías se identificaron 26 tecnologías adoptadas, 7 tecnologías parcialmente adoptadas y 1 tecnología rechazada, los cuales se resumen en un promedio de 83,6\% de tecnologías aplicadas por las familias del Sistema Semi Intensivo, un $68,2 \%$ por parte de las familias del Sistema en Transición y un $24,4 \%$ de las familias del Sistema Extensivo. Conclusiones: El tiempo determinado de cambio tecnológico de las familias campesinas en la comunidad de Huisa Ccollana está relacionado directamente con el grado de mejoramiento del ganado y la producción lechera, donde el mejoramiento por cruzamientos absorbentes tarda en promedio 15 años para lograr una buena producción e insertarse al mercado. En las familias que realizaron el mejoramiento mediante un cambio general del hato de animales criollos por animales mejorados, el cambio tecnológico se dio aproximadamente a los 8 años. La diferencia entre ambas formas de cambio tecnológico fue de 7 años.

Palabras clave: Ganadería extensiva, cambio tecnológico, ganadería semi intensiva

\section{ABSTRACT}

Objective: Describe and analyze the process of technological change in extensive and semi-intensive livestock production systems in the rural community of Huisa Ccollana. The specific objectives were: Identify and characterize the different livestock production systems in the rural community of Huisa Ccollana; identify and analyze internal and external factors that positively and/or negatively influence technological change in livestock production systems; Identify and analyze the process of technological change in livestock production systems. Materials and Methods: For the descriptive analysis of the Technological Change, the following evaluation parameters were used: Observation, Semi-structured Interview and Applied Survey (through the application of a survey, more accurate data and information could be collected). The unit of analysis is the peasant family (family economic unit), the number of samples were 30 families, which represented $25 \%$ of the total families in the community. The percentage of application of technologies by production systems was determined by their evolution by activity (improvement, food, management, health, infrastructure, agribusiness, environment). Results: In the introduction and adoption of technologies, 26 adopted technologies, 7 partially adopted technologies and 1 rejected technology were identified, which are summarized in an average of $83.6 \%$ of technologies applied by the families of the Semi Intensive System, $68.2 \%$ per share. of the families of the System in Transition and $24.4 \%$ of the families of the Extensive System. Conclusions: The determined time of technological change of the peasant families in the community of Huisa Ccollana is directly related to the degree of improvement of the cattle and the milk production, where the improvement by absorbent crosses takes on average 15 years to achieve a good production and enter the market. In the families that made the improvement through a general change of the herd of Creole animals for improved animals, the technological change occurred at approximately 8 years. The difference between both forms of technological change was 7 years.

Keywords: Extensive livestock, technological change, semi-intensive livestock.

\section{INTRODUCCIÓN}

La provincia de Espinar es denominada la capital ganadera de la Región Cusco por la tenencia en cantidad y calidad de ganado, esto debido a que la actividad ganadera es la que brinda mayores ingresos a la familia campesina (Blanco, 1987; Figueroa, 1987; Gonzales de Olarte, 1989). La comunidad campesina de Huisa Ccollana es una de las pocas comunidades pioneras en la crianza tecnificada de vacunos lecheros a nivel provincial (Casallo et al., 2000; Centro de Formación Campesina, 2001). En estos últimos años las familias productoras de esta comunidad, están viviendo un proceso de cambio de su sistema de crianza, adoptando diferentes técnicas, métodos y tecnologías propios de un manejo adecuado de los animales (Centro de Formación
Campesina, 2001). Para dicho cambio intervienen diferentes factores internos y externos que influyeron e influyen, estos factores de cambio que son indispensables para el desarrollo de la producción animal, han tenido que pasar por un proceso largo en términos de tiempo y espacio, los cuales están siendo manejados en forma sistémica reduciendo sus limitantes (Tapia, 1984). Las familias campesinas se dan cuenta que aprovechando de mejor manera sus recursos mínimos (tierra, agua, pasto, semovientes y trabajo), interactuando con otros factores internos y externos y enfocando la actividad agropecuaria como un SISTEMA, están logrando resultados positivos que se traducen en mayores ingresos y mejor nivel de vida (Murra,1975; Greslou,1986; Gonzales de Olarte, 1989).

Este trabajo de investigación nace de la necesidad de conocer

\footnotetext{
${ }^{1}$ Facultad de Agronomía y Zootecnia. Universidad Nacional de San Antonio Abad del Cusco.

${ }^{2}$ Escuela de Posgrado. Universidad Nacional José Faustino Sánchez Carrión.

${ }^{3}$ Facultad de Ingeniería Química y Metalúrgica. Universidad Nacional José Faustino Sánchez Carrión.
} 
el proceso de cambio tecnológico en el sistema de producción ganadera de la comunidad campesina de Huisa Ccollana, para la selección de la comunidad se determinó cuatro variables muy importantes: como la representatividad por ser una comunidad alto andina del sur del Perú, su liderazgo en la producción ganadera, su variabilidad dentro del sistema agropecuario y por ser una zona muy poco estudiada, para lo cual se realizó la recopilación de la información mediante los métodos de observación directa, entrevistas semi estructuradas y las encuestas aplicadas.

\section{MATERIALES Y MÉTODOS}

La caracterización por sistemas de producción ganadera se analizó desde el punto de vista cualitativo cuyas variables de comparación son: el objetivo de la producción, la infraestructura de riego, sistema de pastoreo, el uso de la maquina agrícola, el uso de insumos externos y su relación con el mercado; asimismo se analizó cuantitativamente tomando en consideración las variables como: ubicación de la parcela, tierra, riego (Variables Físicas), grado de instrucción, edad del jefe de familia, organización, ingreso (Variables Socio-Económicas), producción de leche, pasto cultivado, vacuno mejorado, forraje cultivado (Variables de Producción Ganadera) (Plaza y Francke, 1981; Kervyn, 1987; Grillo, 1989).

Para el desarrollo del proceso de cambio tecnológico en los Sistemas de Producción Ganadera, se identificaron los factores que intervienen en el proceso de cambio tecnológico los cuales son: factores internos (tierra, pasto, agua, organización, toma de decisiones, capital fijo); factores externos (capacitación, crédito, vías de comunicación, medio ambiente y mercado) (Alagón et al., 1996; Apollin y Eberhart, 1999).

Para la selección de la comunidad de Huisa Ccollana, provincia de Espinar, departamento del Cusco se tomaron en cuenta las siguientes variables:

Representatividad por ser parte de las comunidades campesinas alto andinas del sur del Perú.

Liderazgo en la crianza tecnificada de ganado vacuno de producción lechera (Brown Swiss).

Variabilidad en los sistemas de producción Agropecuaria.

Inexistencia de estudios en dicha zona

Tabla 1. Sistema de producción ganadera familiar.
Para la recolección de la información se utilizaron los siguientes métodos:

Método de Observación: Que consistió en realizar con el sentido de la vista las observaciones del sistema de crianza y vivencia de las familias campesinas.

Método de Entrevista semi-estructurada: Que consistió en realizar las visitas y diálogos a una o a un grupo de personas, para ello se formuló anticipadamente preguntas sobre el tema acerca del cual interesa conocer las opiniones y criterios de los actores.

Método de Encuesta aplicada. Mediante la aplicación de una encuesta se pudo recoger datos e información más precisa.

La recolección de información complementaria se realizó mediante la revisión de documentos bibliográficos acerca de la comunidad en estudio como: padrón comunal, libro de actas, informes históricos y artículos científicos.

La unidad de análisis es la familia campesina (unidad económica familiar). El número total de familias fue de 120, el tamaño de muestra es el $25 \%$ del total de las familias de la comunidad el cual representa a 30 familias de la comunidad. Para la caracterización de los sistemas de producción ganadera, se analizó mediante el enfoque de sistemas, su estructura, su funcionalidad y sus elementos; de ese razonamiento lógico se identificaron los componentes principales que intervienen en la actividad ganadera (Achata, 1990; Sotomayor, 1996), descomponiendo dichos componentes se identificaron las variables e indicadores los cuales se muestran en la Tabla 1.

Para la tipificación de los sistemas de producción ganadera, se realizó la recolección de la información primaria identificando diferentes variables las cuales se procesaron mediante el análisis multivariable lógico identificando 16 variables (Tabla 2) COMPONENTES

\begin{tabular}{|c|c|c|}
\hline \multirow{3}{*}{$\begin{array}{l}\text { RECURSO } \\
\text { FísICO }\end{array}$} & > Recurso tierra & > Estructura, textura, tenencia \\
\hline & > Recurso agua & > Áreas con riego \\
\hline & Pastos naturales & > Has \\
\hline \multirow{5}{*}{$\begin{array}{l}\text { SOCIO } \\
\text { ECONÓMICO }\end{array}$} & > Grado de instrucción & > Grado de estudios \\
\hline & > Fuerza de trabajo familiar & $>\mathrm{N}^{\circ}$ de actividades agropecuarias por miembro \\
\hline & > Migración & > Servicio/meses \\
\hline & > Organización & Junta directiva y comités \\
\hline & $>$ Ingreso familiar & Soles mensuales \\
\hline \multirow{5}{*}{$\begin{array}{l}\text { PRODUCCIÓN } \\
\text { GANADERA }\end{array}$} & Alimentación & Tenencia de alimento \\
\hline & > Mejoramiento & > Tenencia de ganado \\
\hline & > Manejo & > Nivel de mejoramiento animal \\
\hline & > Sanidad & > Técnicas de manejo \\
\hline & & > Prácticas de prevención y tratamiento de \\
\hline
\end{tabular}


Tabla 2. Listado de variables cuantitativas empleadas en la tipificación de los sistemas de producción ganadera familiar.

\begin{tabular}{|c|c|}
\hline $\mathrm{N}^{\circ}$ & Descripción de variables/indicadores \\
\hline 01 & Pasto cultivado, hectáreas \\
\hline 02 & Vacunos mejorados, unidades \\
\hline 03 & Tierras con riego, hectáreas \\
\hline 04 & Forraje cultivado, hectáreas \\
\hline 05 & Producción de leche, litros \\
\hline 06 & Praderas mejoradas, hectáreas \\
\hline 07 & Vacunos cruzados, unidades \\
\hline 08 & Área total familiar, hectáreas \\
\hline 09 & Tenencia de ganado vacuno, unidades \\
\hline 10 & Tenencia de ganado ovino, unidades \\
\hline 11 & Tenencia de camélidos, unidades \\
\hline 12 & Vacunos criollos, unidades \\
\hline 13 & Pasto natural, hectáreas \\
\hline 14 & Grado de instrucción (primaria, secundaria) \\
\hline 15 & Edad del jefe de familia, años \\
\hline 16 & Tierras sin riego, hectáreas \\
\hline
\end{tabular}

\section{RESULTADOS Y DISCUSIÓN}

En nuestras descripciones y análisis se pudo observar tres tipos de sistemas de producción ganadera familiar existente en la comunidad de Huisa Ccollana, los cuales se codificaron como T1= Sistema de Producción Ganadera Semi Intensiva; T2=Sistema de Producción Ganadera en Transición y T3= Sistema de Producción ganadera extensiva (tabla3).

Tabla 3. sistemas de producción ganadera familiar en la comunidad.

\section{CODIGO}

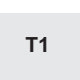

T2

T3

T1 Sistema de Producción Ganadera Semi Intensiva

Sistema de Producción Ganadera en Transición $58 \%$ Sistema de Producción Ganadera Extensiva

$35 \%$

$100 \%$

TOTAL
FAMILIAS
8

70

42

120
La caracterización de los sistemas de producción ganadera familiar en la comunidad campesina de Huisa Ccollana la describimos en la Tabla 4, en la cual se puede observar que existen 7 variables de comparación: objetivo de la producción, riego e infraestructura, sistemas de pastoreo, producción agropecuaria, uso de maquinaria, uso de insumos externos y relación con el mercado.

El sistema de producción ganadera extensiva se caracteriza por que ordena el territorio y los medios de producción en la lógica de una economía tradicional y simple, donde la producción mayormente depende de las lluvias, donde los medios físicos son explotados de acuerdo a la oferta natural, siendo tierras de baja calidad, cuyos rebaños son pastoreados en grandes extensiones de pastos naturales pobres y frágiles, presentando rebaños de baja calidad y rendimiento, existiendo un nivel bajo de conocimientos para la producción y escasa introducción de tecnologías. En la Tabla 5 se detalla algunas variables importantes ya que agrupa a familias campesinas que poseen un 0.67 Has en promedio de pastos cultivados, con 2.2 Has de tierras con riego, un capital pecuario de 3.8 vacunos cruzados, 8 vacunos criollos, un promedio de 21 ovinos y 5 animales entre camélidos y equinos, el ingreso familiar promedio fue de 237.65 nuevos soles mensuales (67.9 dólares) por la venta de sus animales criollos, esto significa que no satisface sus necesidades mínimas lo que obliga a realizar trabajos secundarios para cubrir sus necesidades de alimentación y transporte.

El sistema de producción ganadera en transición se caracteriza por estar en un proceso de acción de pasar de un estado de producción a otro diferente, con dirección a la semi intensificación, es un sistema basado en el reordenamiento territorial, el reacondicionamiento de los medios físicos donde la producción depende de un mejor aprovechamiento de los recursos físicos (fertilización de suelos, canales de irrigación, mejoramiento de praderas, etc.). Existe la introducción y adopción de nuevos conocimientos e introducción de nuevas tecnologías, existiendo un cambio en el agro ecosistema y el rebaño cuantitativa y cualitativamente. Existe mayor demanda de tecnologías y conocimientos, los comuneros combinan conocimientos y recursos de un sistema de producción extensiva tradicional y tecnologías para la intensificación productiva. Existe una creciente seguridad alimentaria familiar ya que la producción está siendo orientada al mercado.

En la Tabla 5 se detalla algunas variables importantes ya que agrupa a familias campesinas que poseen 3,6 Has en promedio de pastos cultivados, con 6,9 Has de tierras con riego, con un capital pecuario de 15 vacunos mejorados, el ingreso familiar promedio fue de 762,3 nuevos soles mensuales $(217,8$ dólares), por lo tanto, dicho ingreso es reinvertido en la actividad ganadera, en su mayoría son comuneros con experiencia media, participan activamente en el desarrollo de su comunidad, están en constante capacitación y tienen bien claro hacia dónde quieren orientar su producción.

El sistema de producción ganadera semi intensiva se caracteriza por el ordenamiento territorial y los medios de producción en la lógica de una economía de mercado, donde es definido el tipo de producción, adoptando conocimientos y tecnologías, existiendo mejoras cuantitativas y cualitativas en el agro ecosistema y el rebaño familiar, presenta un bajo gasto energético debido a un manejo racional en términos de tiempo y espacio de la crianza ya que el pastoreo se realiza en áreas cercanas a sus viviendas combinando con otras formas de alimentación (heno, ensilado).

La producción se acrecienta dando seguridad y beneficios a la familia ya que la producción está orientada al mercado. En la Tabla 5 se detalla algunas variables importantes ya que agrupa a familias campesinas que disponen de mayores tierras de pastos cultivados 5,4 Has en promedio, con 11,8 Has de tierras con riego, con un capital pecuario de 21 vacunos mejorados, el ingreso familiar promedio fue de 1403,7 nuevos soles mensuales (401,07 dólares), por lo tanto tiene mejores niveles de vida con respecto a los otros grupos de familias, también reinvierten en la ganadería, en su mayoría son comuneros con mucha experiencia, participan activamente en el desarrollo de su comunidad, y son visitados constantemente mediante pasantías, intercambio de experiencias por otros comuneros de otras regiones del país, para que vean in situ el desarrollo de sus crianzas, asimismo el precio de sus animales tiene alto valor por su mejoramiento y manejo tecnificado. 
Tabla 4. Cuadro comparativo cualitativo de los sistemas de producción ganadera.

\section{VARIABLES DE} COMPARACIÓN

SISTEMA DE PRODUCCIÓN EXTENSIVO

Optimizar escasos recursos, autoconsumo, trueque y pequeños excedentes para el mercado. Mercantil simple.

\begin{tabular}{ll} 
1. Objetivo de la producción & $\begin{array}{l}\text { Optimizar escasos recursos, autoconsumo, } \\
\text { trueque y pequeños excedentes para el } \\
\text { mercado. Mercantil simple. }\end{array}$ \\
\hline 2. Riego e infraestructura & Riego temporal, escasa infraestructura.
\end{tabular}

3. Sistema de pastoreo Extensivo, pequeños rebaños de baja calidad pastoreados en grandes extensiones de praderas naturales pobres y frágiles, su sistema praderas naturales pobres y frágl.
de pastoreo es rotativo estacional.

4. Producción agropecuaria.

Cultivos tradicionales de auto consumo y estrecha articulación con ganadería criolla de pastoreo extensivo.

5. Uso de maquinaria

Escasa

6. Uso de insumos externos: Muy poco semillas, abonos,

productos veterinarios $y$

otros.

7. Relación con el mercado. Muy poca y esporádica

Tabla 5. Resumen comparativo cuantitativo de los sistemas de producción ganadera.
SISTEMA DE PRODUCCIÓN EN TRANSICIÓN

Optimizar y mejorar la calidad de los recursos y patrimoni

Infraestructuras de riego e inicio de innovaciones tecnológicas bajo riego.

Introducción de pastos cultivados y conservación de forrajes el pastoreo se realiza en las praderas nativas combinando con los pastos cultivados y conservados. Sistema rotativo fraccionado.

Incorporación de nuevos cultivos bajo riego y ganado mejorado, interacciones muy fuertes entre

Mediana: solo para actividades claves de roturación y preparación de tierras (tractor y equipos)

Introducción de semillas mejoradas y en algunos casos abono con tendencia a lo orgánico, introducción de productos veterinarios
SISTEMA DE PRODUCCIÓN SEMI INTENSIVA

Mercado: maximizar la ganancia, lógica de economía de mercado

Utilización de sistemas de riego adecuados

a alimentación es a base de pastos cultivados y con forrajes conservados combinados con las raderas mejoradas, el sistema de pastoreo es rotativo fraccionado.

Optimización y adopción de los cultivos bajo riego pariog gana serí estabulados, con animales PPC, componentes.

Alta: Para actividades claves de roturación y preparación de tierras (tractor y equipos)

Intensivo: semillas, abonos orgánicos (humus, compost), productos veterinarios.

Alta

\begin{tabular}{|c|c|c|c|}
\hline VARIABLES & EXTENSIVO & TRANSICIÓN & SEMI INTENSIVO \\
\hline $\begin{array}{l}\text { 1. FísICO } \\
\text { 1.1 Ubicación de parcela. } \\
\text { 1.2 Tierras con riego (has) } \\
\text { 1.3 Tierras sin riego (has) } \\
\text { 1.4 Pasto natural (has) } \\
\text { 1.5 Área total familiar (has) }\end{array}$ & $\begin{array}{c}\text { Parte alta } \\
2,2 \\
18,8 \\
199 \\
21\end{array}$ & $\begin{array}{c}\text { Parte baja } \\
\quad 6,9 \\
7,8 \\
9,2 \\
15\end{array}$ & $\begin{array}{l}\text { Parte baja } \\
11,8 \\
8,2 \\
9,7 \\
20\end{array}$ \\
\hline $\begin{array}{l}\text { 2. } \\
1.1 \quad \text { GOCIO ECONÓMICO } \\
1.2 \quad \text { Edad de jefstrucción. } \\
1.3 \quad \text { Organización } \\
\text { Ingreso familia (años) }\end{array}$ & $\begin{array}{l}4 \text { prim. } \\
\quad 41 \\
\text { Baja } \\
\$ 67,9\end{array}$ & $\begin{array}{l}5 \text { prim. } \\
43 \\
\text { Alta } \\
\$ 217,8\end{array}$ & $\begin{array}{l}6 \text { prim. } \\
48 \\
\text { Alta } \\
\$ 401,7\end{array}$ \\
\hline $\begin{array}{l}\text { 3. PRODUCCIÓN } \\
\text { 3.1 Producción de leche (litros) } \\
\text { 3.2 Pasto cultivado (ha) } \\
\text { 3.3 Vacunos mejorados } \\
\text { 3.4 Forraje cultivado (ha) } \\
\text { 3.5 Vacunos criollos (unidades) } \\
\text { 3.6 Vacunos cruzados (unidades) } \\
\text { 3.7 Tenencia de unidades vacuno(unid) } \\
\text { 3.8 Tenencia de unidades ovino (unid) } \\
\text { 3.9 Tenencia de unidades de camélidos (unidades) } \\
\text { 3.10 Praderas mejoradas (has) }\end{array}$ & $\begin{array}{l}5 \mathrm{Lt} / \mathrm{dia} \\
0,67 \mathrm{Ha} \\
1,5 \% \\
0,2 \mathrm{Ha} \\
0 \\
0 \\
13 \\
21 \\
5 \\
0,2\end{array}$ & $\begin{array}{c}15 \mathrm{Lt} \\
3,6 \mathrm{Ha} \\
97,5 \% \\
1,3 \mathrm{Ha} \\
0,6 \% \\
1,8 \% \\
16 \\
13 \\
1 \\
0,8\end{array}$ & $\begin{array}{l}34,4 \mathrm{Lt} \\
5,4 \mathrm{Ha} \\
100 \% \\
2,1 \mathrm{Ha} \\
69,3 \% \\
29,2 \\
22 \\
3 \\
0 \\
2,8\end{array}$ \\
\hline
\end{tabular}

Fuente: Encuestas, Entrevistas semi estructuradas 2002.

Los factores que intervinieron en el proceso de cambio tecnológico fueron factores favorables y factores limitantes (Tabla 6), para la identificación de los factores de cambio tecnológico en la comunidad se tomó información de sondeos y de las encuestas semi estructuradas.

Tabla 6. Factores favorables y limitantes en el proceso de cambio tecnológico.

\begin{tabular}{|c|c|c|c|}
\hline \multicolumn{2}{|c|}{ FACTORES FAVORABLES } & \multicolumn{2}{|c|}{ FACTORES LIMITANTES } \\
\hline Internos & Externos & Internos & Externos \\
\hline $\begin{array}{l}\text { Organización } \\
\text { Toma de decisiones } \\
\text { Recurso tierra } \\
\text { Recurso agua } \\
\text { Recurso pasto } \\
\text { Capital fijo }\end{array}$ & $\begin{array}{l}\text { Capacitación } \\
\text { Crédito } \\
\text { Vías de } \\
\text { comunicación } \\
\text { Mercado } \\
\text { Medio Ambiente }\end{array}$ & $\begin{array}{l}\text { Recurso tierra } \\
\text { Escases de recurso } \\
\text { agua } \\
\text { Débil organización } \\
\text { Conformismo }\end{array}$ & $\begin{array}{l}\text { Escaso apoyo } \\
\text { institucional } \\
\text { No existencia de } \\
\text { crédito } \\
\text { Medio Ambiente } \\
\text { desfavorable }\end{array}$ \\
\hline
\end{tabular}




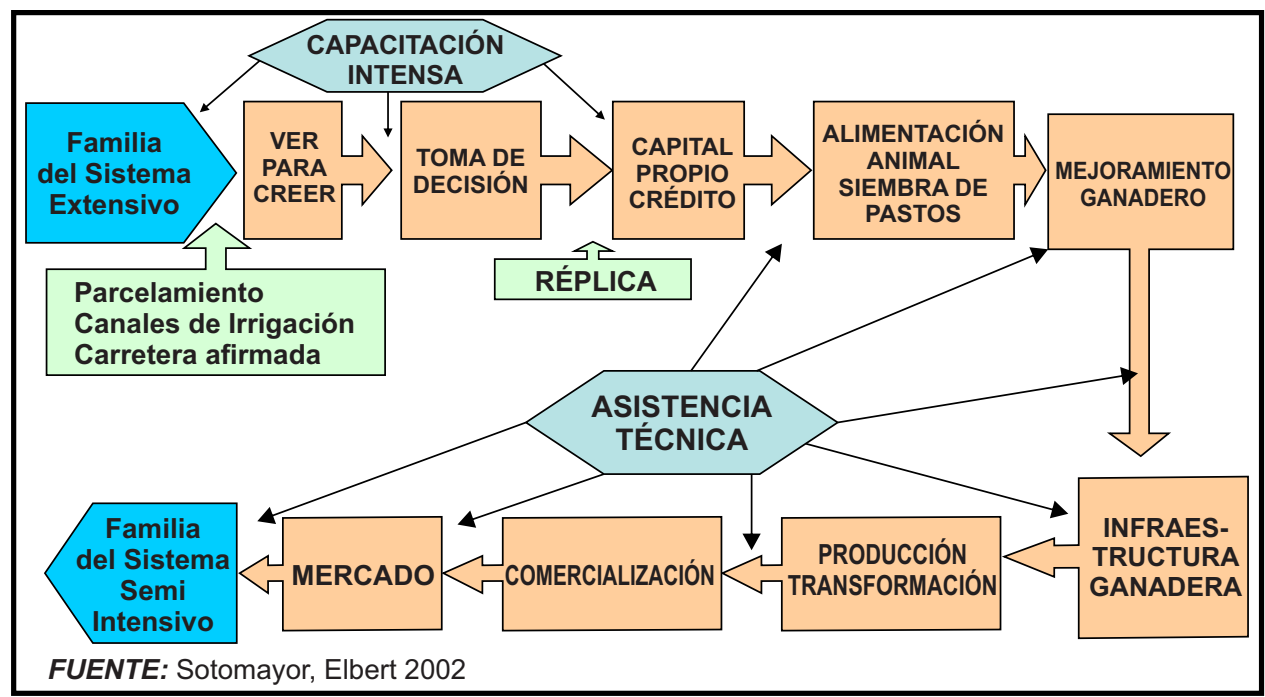

Figura 1. Esquema del proceso de cambio tecnológico a nivel familiar.

En la figura 1 se puede observar CAMBIO tecnológico a nivel familiar, como una familia del tipo extensivo con una crianza tradicional no tecnificada ha desarrollado su sistema de crianza diferentes acciones que han sido fundamentales para el cambio en su tecnología de crianza. Primeramente las familias han tenido que ver para creer, mediante las demostraciones en pequeñas parcelas de la producción de patos y otros, bajo condiciones de la Provincia de Espinar por parte de las Organizaciones No Gubernamentales (ONGs), asimismo han teniendo que ver a comunidades vecinas con similares características ecológicas que la de Espinar, como las comunidades del Departamento de Puno (Achata, 1990), y una vez convencidos de que en las condiciones en que están se pueden producir, han tenido que tomar una decisión, de cambiar su sistema de crianza y de vida, han tenido que buscar las instituciones crediticias para que puedan tener un capital para empezar o realizar la venta de algunos semovientes que poseías, las instituciones crediticias fueron el Banco Agrario, Proyecto Pachacutec, las pocas ONGs que existían y existen. Con ese pequeño capital se preocuparon por la alimentación animal, sembrando los pastos cultivados, seguidamente mejorando su ganado mediante los cruzamientos absorbente con ganados mejorados de la raza Brown Swiss de temperamento lechero, mejorando sus infraestructuras ganaderas, al cabo de algunos años llegaron a producir mediante el manejo constante y tecnificado, transformando los productos y llevando al mercado regional y convirtiéndose en familias ganaderas productoras semi intensivas.

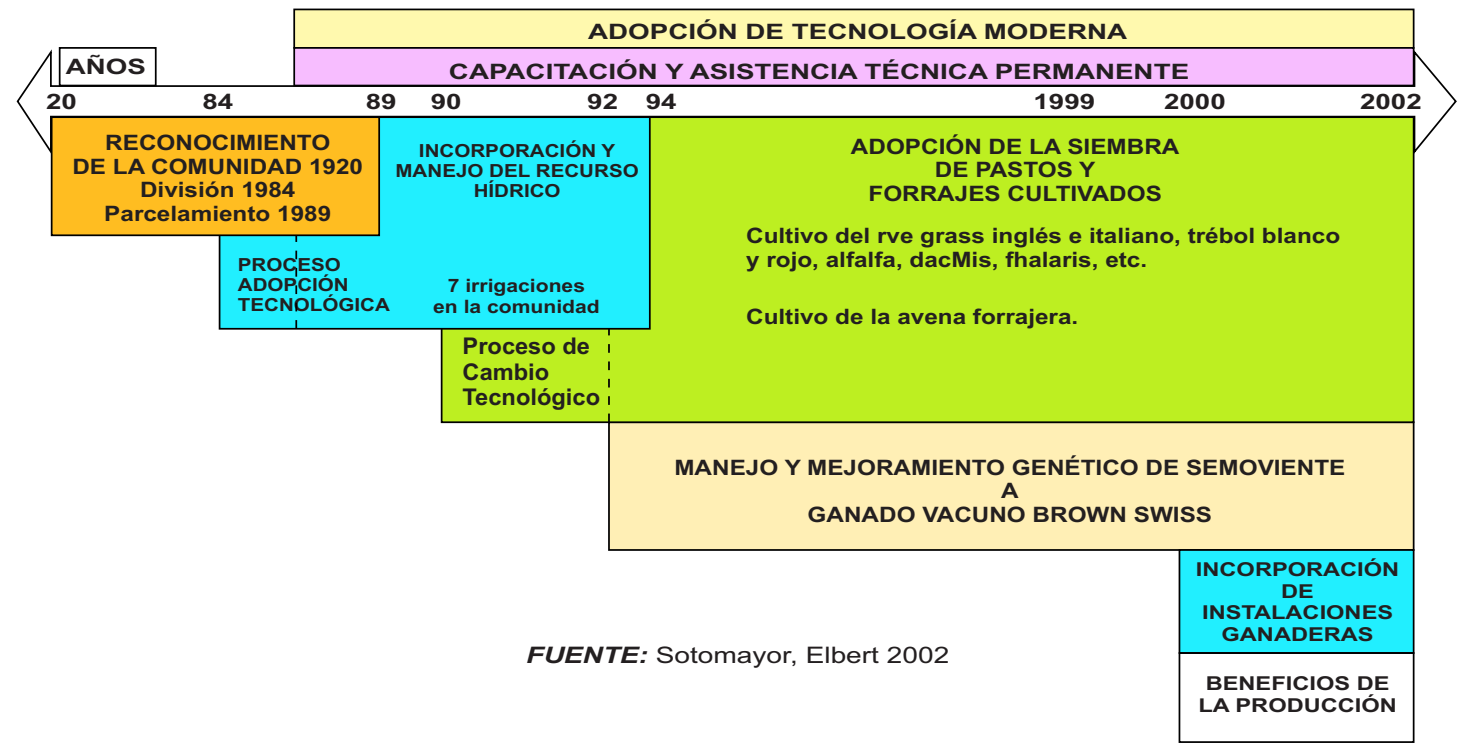

Figura 2. Esquema del proceso de cambio tecnológico a nivel comunal con mejoramiento mediante el cruzamiento absorbente

En la Figura 2 se puede observar el proceso de CAMBIO TECNOLÓGICO A NIVEL COMUNAL, en este proceso se realizaron muchas actividades, siendo las más importantes: el RECONOCIMIENTO, DIVISIÓN y PARCELAMIENTO de la tierra que término el año 1989. A partir de ese año las familias campesinas se preocuparon por desarrollar sus RECURSOS HÍDRICOS, logrando construir 7 canales de irrigación y un comité de regantes hasta el 1994, seguidamente realizaron la siembra de pastos y forrajes desde 1990, luego el mejoramiento genético de los animales, la incorporación de instalaciones ganaderas, produciendo y transformando el producto leche, llevando productos al mercado y sacando beneficios de la producción, eso se mantiene en la actualidad. 


\section{CAPACITACIÓN Y TRABAJO CONSTANTE}

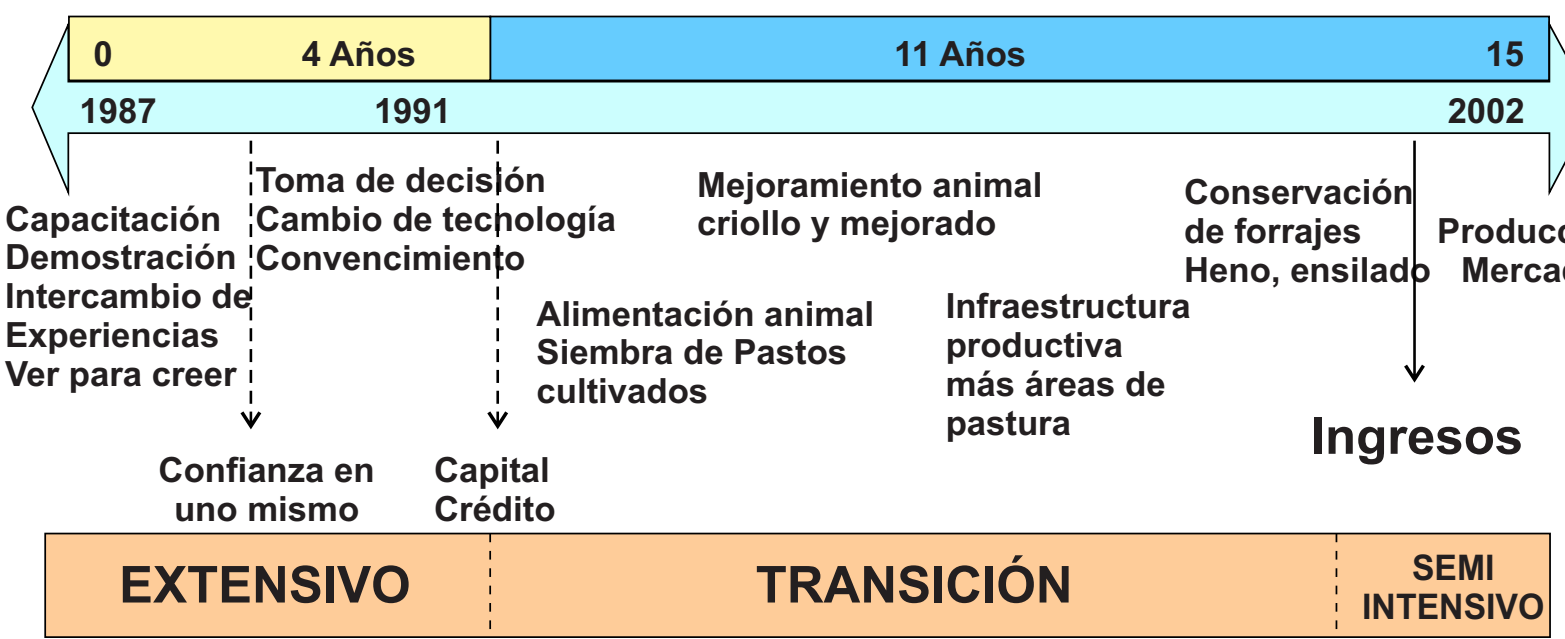

Figura 3. Tiempo determinado de cambio tecnológico en las familias campesinas

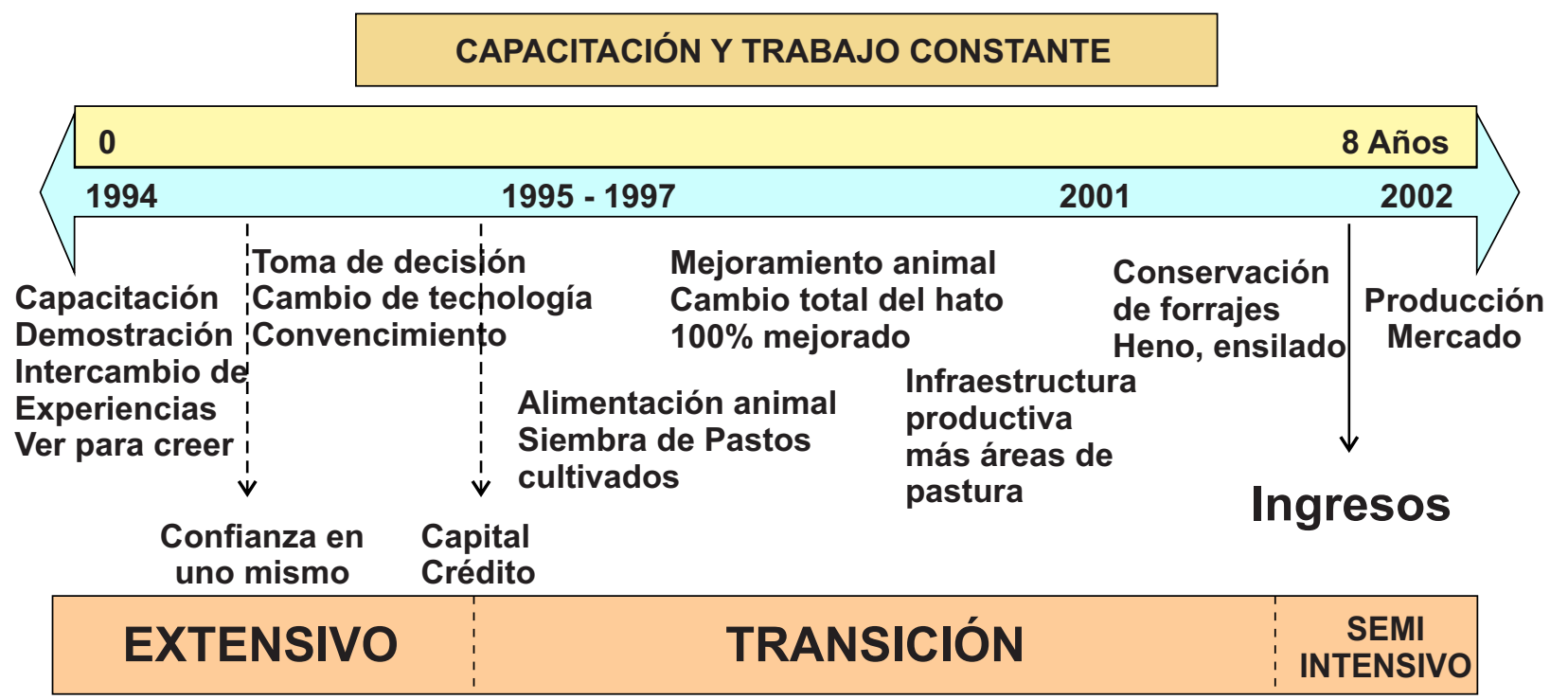

Figura 4. Tiempo determinado de cambio tecnológico en las familias campesinas con el cambio total del hato ganadero.

En las Figuras 3 y 4 , se puede observar que el tiempo determinado de cambio tecnológico tuvo una relación directa con el grado de mejoramiento de los animales lecheros.

En la Figura 3 se grafica el periodo determinado con un mejoramiento de los animales mediante los cruzamientos absorbentes y se tiene un promedio de15 años para lograr una buena producción, estar en las mismas condiciones de las familias del T1 (semi intensivas); en la Figura 4 se detalla el tiempo determinado con un cambio general del hato de animales criollos por animales mejorados con producción, el tiempo que transcurrió para llegar a estar en las mismas condiciones que de las familias del T1 es un aproximado de 8 años, la diferencia entre ambas formas de cambio tecnológico fue de 7 años. Algunas familias de la comunidad de Huisa Ccollana ahorraron 7 años en alcanzar niveles de desarrollo semi intensivo.

\section{CONCLUSIONES}

Se identificaron 3 sistemas de producción ganadera en las familias campesinas: Sistema de producción ganadera semi intensivo (T1); Sistema de producción ganadera en transición (T2) y Sistema de producción ganadera extensivo (T3).

Los factores internos que influyeron favorablemente en el proceso de cambio tecnológico son: tierra, organización, agua, toma de decisión, pasto, capital semoviente. Los factores externos que influyeron favorablemente en el proceso de cambio tecnológico son: capacitación, crédito, vías de comunicación, mercado, medio ambiente.

Los factores internos que limitan el cambio tecnológico en las familias del sistema de producción extensiva (T3) son: tierra (ubicación de sus terrenos en las partes altas), escasez de agua, desorganización, conformismo. Los factores externos que limitan el cambio tecnológico en las familias del sistema de producción extensiva (T3) son: escasa capacitación, crédito nulo, medio ambiente desfavorable.

El inicio del cambio tecnológico, de su sistema de crianza y modo de vida fue a partir de la parcelación y propiedad de la tierra; seguidamente se fortaleció la organización, la toma de 
decisión (trabajo), el manejo de los recursos naturales como el agua, la tierra, el pasto, los semovientes, luego la mejora de las vías de comunicación, el crédito y la inserción al mercado.

\section{RECOMENDACIONES}

Apoyar a las familias del sistema de producción extensivo (T3) con la mejor utilización del recurso agua (riego por aspersión con reservorios rústicos), además ampliar el cultivo de avena ya que es un cultivo estacional.

Mediante este trabajo apoyar a las comunidades vecinas cuyas características son similares a las de Huisa Ccollana. Realizar similares trabajos en distintas zonas para poder tener un banco de información y poder contribuir en forma real y técnica en el desarrollo rural.

Dar prioridad y una mayor capacitación en comercialización de productos de origen pecuario.

Fomentar y propiciar políticas reales y efectivas de asistencia crediticia y técnica, orientadas al segmento del campesinado del estrato social: pobre y muy pobre

\section{REFERENCIA BIBLIOGRAFICA}

Achata, A. (1990). Propuestas de Metodologías de investigación de los Sistemas de Producción Agrícola en la Comunidad de Puno. INIA-PISA-Puno. Perú.

Alagón H., A; Agramonte V., M; Visaga A., R. (1996). Sistemas de Producción y de Alimentación del ganado en las Comunidades Campesinas de la Cuenca de Pomacanchi. Gobierno Regional Inka, Instituto de Manejo de Agua y Medio Ambiente - IMA, Cusco, Perú.

Apollin, F; Eberhart, C. (1999). Análisis y Diagnóstico de los Sistemas de Producción en el Medio Rural. Guía Metodológica. Sistema de capacitación para el manejo de los Recursos Naturales Renovables CAMAREN, Coordinación CARE, CESA, IEDECA, RAFE, Quito, Ecuador.

Blanco, O; (1987). Domesticación y Mejoramiento Genético en los Andes. Cusco, Perú.

Casallo, L; Ibañez, N; Ochoa, R; Rodriguez, P; Stewart, S. (2000). Diagnóstico de la Provincia de Quispicanchi 2000. Centro de Capacitación Agro Industrial Jesús Obrero-CCAIJO, Cusco, Perú.
CENTRO DE FORMACION CAMPESINA - CFC - DE LA PRELATURA DE SICUANI. 2001. Sistematización de experiencias sobre la producción, manejo y conservación de pastos y forrajes en la Provincia de Espinar. Yauri. Cusco, Perú.

Figueroa, A. (1987). La economía Campesina de la Sierra del Perú. PUCP. Fondo Editorial. Lima, Perú.

gonzales de Olarte, E. (1989). Economía de la comunidad Campesina. Instituto de Estudios Peruanos. Lima, Perú.

Greslou, F; Ney, B. (1986). Un Sistema de Producción Andino: El Caso de los Comuneros de San Juan y Huascoy - Valle de Chancay. Instituto Francés de Estudios Andinos - IFEA y Centro de Estudios Rurales Andinos "Bartolomé de las Casas" - CBC, Cusco, Perú.

Grillo, E. (1989). Sistematización de la Tecnología Agraria en el Contexto del desarrollo Rural de la Sierra Peruana. INP-DSE.FELDAPING. Alemania.

Kervyn, B. (1987). La Organización del Espacio en Comunidades de la Sierra Sur del Perú. CERA. Cusco, Perú.

Murra, Y. (1975). Formaciones Económicas y políticas del Mundo Andino. Instituto de Estudios Peruanos - IEP. Lima. Perú.

Plaza, O; Francke, M; (1981). Formas de Dominio, Economía y Comunidades Campesinas. Centro de Estudios y Promoción del Desarrollo - DESCO. Lima, Perú.

Sotomayor, W. (1997). Caracterización de Sistemas de Producción Agroecológica Tradicional en Comunidad Campesina y Evaluación de Sostenibilidad e Impacto Ecológico Medio Ambiental. Apurímac, Perú.

Tapia, M. (1984). Pastoreo y Pastizales de los Andes del Sur del Perú. Instituto Nacional de Investigación y Promoción Agropecuaria. Lima, Perú. 